prevalence of low flow and its accompanying vascular functional parameters after successful aortic valve replacement (AVR).

Aim of the study and methods We set out to assess the prevalence of low flow as well as of abnormal valvulo-aortic impedance and systemic arterial compliance following AVR. We recruited unselected, consecutive patients attending our echocardiography laboratory who had interpretable echo images and LF ejection fraction $>\geq 50 \%$. We calculated aortic valve area (AVA) by continuity equation; stroke volume indexed to body surface area $\left(\mathrm{SVi}=\mathrm{LVOT}_{\mathrm{VTI}} \mathrm{x} \mathrm{LVOT}_{\text {Area }}\right.$; units $-\mathrm{ml} /$ $\mathrm{m}^{2}$; normal $>35 \mathrm{ml} / \mathrm{m}^{2}$ ) valvulo-aortic impedance $\mathrm{Zva}=$ $(\mathrm{MPG}+\mathrm{SBP}) / \mathrm{SVi})$; units - $\mathrm{mmHg} / \mathrm{ml} / \mathrm{m}^{2}$; normal $<5 \mathrm{mmHg} /$ $\mathrm{ml} / \mathrm{m}^{2}$ where MPG is mean pressure gradient, SBP is systolic blood pressure and systemic arterial compliance $(\mathrm{ml} / \mathrm{mm} \mathrm{Hg}$ / $\mathrm{m}^{2}$ ) SAC $=\mathrm{SVi} /$ Pulse pressure), normal $<\leq 0.6$.

Results We studied 77 patients with AVR, 49 male, mean age (SD) 68 (7.8) years. The mean AVA (SD) was 1.59 (0.59) $\mathrm{cm}^{2}$; mean Zva (SD) was $5.02(0.58)$; mean SVi (SD) was 30.6 (9.6); mean SAC was $0.48(0.18)$. AVA was $<1 \mathrm{~cm}^{2}$ in 11 (14\%) patients with AVA, $1-1.5 \mathrm{~cm}^{2}$ in $24(32 \%)$ and $>1.5 \mathrm{~cm}^{2}$ in $424(55 \%)$. SVi was low in $57(74 \%)$, Zva was elevated in $37(48 \%)$, and SAC was elevated in $16(21 \%)$ of patients. A higher proportion of patients had abnormal SVi, Zva and SAC amongst those with AVA $<1.5 \mathrm{~cm}^{2}$ than in those with AVA $>1.5 \mathrm{~cm}^{2}$.

Conclusion A significant proportion of patients have abnormal valvulo-aortic loads, low-flow states, and abnormal arterial compliance after successful aortic valve replacement. Further study is warranted to assess the potential clinical significance of these findings.

\section{EARLY EVOLUTION OF A MINIMALLY INVASIVE MITRAL PROGRAMME}

Sion Jones*, Ken Palmer, Omar Al-Rawi, Paul Modi. Liverpool Herat and Chest Hospital; *Presenting Author

\subsection{6/heartjnl-2016-309890.154}

Introduction Minimally invasive mitral valve (MIMV) surgery is technically challenging with a long learning curve. We examined the evolution of repair techniques in a new program. Methods MIMV surgery at our institution is performed through a $6 \mathrm{~cm}$ right anterior minithoracotomy. We examined prospectively collected data on all patients undergoing MIMV between March 2011 and December 2014. Data are presented as medians (interquartile range). Results 99 patients had MIMV procedures during this period. Median age was 61 (53 to 68$)$ years, 31 (31\%) were female, 2 were reoperations. The repair rate for degenerative and functional disease was $94 \%$ (84 of 89 patients) with no/trivial residual MR $(\mathrm{n}=82$, $98 \%)$ or mild MR ( $\mathrm{n}=2,2 \%)$ on the intra-operative echo. All rheumatic valves were electively replaced. Twenty (20\%) patients underwent concomitant procedures: cryomaze $(\mathrm{n}=$ 13), patent foramen ovale closure $(n=6)$, tricuspid valve repair $(\mathrm{n}=3)$. Bypass and clamp times were 196 (170 to 221) and 132 (111 to 150) minutes respectively. There was one conversion to sternotomy for mild residual MR found after femoral decannulation; this patient left hospital 6 days later with no regurgitation. There was no mortality and no strokes. Duration of ventilation, ITU and hospital stay were 8 (6-10) hours, 2 (1-3) and 6 (5-8) days respectively. When examined by tertile, the use of GoreTex loops increased significantly as the programme progressed $(p<0.001)$ while resective techniques declined $(\mathrm{p}<0.001)$. Conclusions High rates of valve repair are achievable with minimally invasive mitral surgery. There has been a significant change in the repair techniques employed as the programme developed.

\section{HISTOPATHOLOGICAL STUDY OF 62 SUDDEN CARDIAC DEATH VICTIMS WITH ISOLATED MITRAL VALVE PROLAPSE}

${ }^{1}$ Alexandros Steriotis*, ${ }^{2}$ Lydia Krexi, ${ }^{1}$ Agata Plonczak, ${ }^{1}$ Gherardo Finocchiaro, ${ }^{1}$ Michael Papadakis, 'Sanjay Sharma, ${ }^{1}$ Mary Sheppard. 'St Georges University Hospital; ${ }^{2}$ Aristotle University of Thessaloniki; * Presenting Author

\subsection{6/heartjnl-2016-309890.155}

The aim of the study was to evaluate the cardiac histopathological findings of sudden cardiac death victims with isolated mitral valve prolapse. We studied 62 sudden cardiac death victims with isolated mitral valve prolapse that represented $1.7 \%$ of our total sudden cardiac death cohort. The heart was examined macroscopically and microscopically. Toxicology results were negative. The series included 33 males and 29 females (mean age $37 \pm 14$ years). In all cases the mitral valve exhibited degenerative changes of bileaflet thickening, stretching and prolapse of the leaflets in to the left atrium. The left ventricle showed no evidence of fibrosis macroscopically, but in 46 (74\%) of cases myocardial fibrosis was detected with histology. The majority of the sudden cardiac death victims with myocardial fibrosis had involvement of both papillary muscles and the adjacent myocardial wall (30 cases, 65\%), while in the remaining it was localised to one papillary muscle and the adjacent wall. The posteromedial papillary muscle and posteroinferior wall were involved in the majority (42 cases, 91\%). The involvement of the left ventricular wall was always subendocardial and/or midmural and the type of fibrosis was replacement type and/or interstitial. In conclusion, the majority of sudden cardiac death with isolated mitral valve prolapse exhibits a bileaflet mitral valve prolapse with localised fibrosis, involving one or both papillary muscles and the adjacent subendocardial layer of the left ventricular wall. This myocardial fibrosis can provide a substrate for ventricular arrhythmias and sudden cardiac death. However 26\% of the sudden cardiac death victims had histologically normal heart so other mechanisms to explain the sudden death must be considered.

\section{PATIENT REPORTED OUTCOMES MEASURES FOLLOWING MITRAL VALVE REPAIR: MINIMALLY INVASIVE VIDEO-ASSISTED COMPARED TO STERNOTOMY}

${ }^{1}$ Jennifer Whiteley, ${ }^{2}$ Sion Jones, 'Klare Exarchou, ${ }^{2}$ Ken Palmer, 'Paul Modi*. 'Liverpool Heart and Chest Hospital; ${ }^{2} \mathrm{LHCH} ;{ }^{*}$ Presenting Author

\subsection{6/heartjnl-2016-309890.156}

Objectives There is a paucity of evidence regarding patient reported outcomes following mitral valve repair (MVP). Our aim was to compare these in patients having minimally invasive (MI) andsternotomy (St) approaches. 